

\title{
Variaciones de la idea de nación y patria en los textos escolares de Historia de Nicaragua. 1871-1930: El arduo camino en la construcción del sentimiento de de nacionalidad nicaragüense
}

\author{
Mtro. Guillermo Fernández Ampié \\ Pitágoras 340 - 4 Colonia Narvarte. Delegación Benito \\ Juárez. México D.F. C. P. 03020 \\ Teléfono (55) 56-38-02-87 \\ Correo Electrónico: gfernam@gmail.com \\ Programa de Doctorado del Posgrado en Estudios \\ Latinoamericanos. \\ Facultad Filosofía y Letras. Universidad Nacional \\ Autónoma de México.
}




\section{Introducción}

Es muy probable que en las naciones centroamericanas, y particularmente en Nicaragua, más que en cualquier otro sea fácil trazar los senderos que ha seguido el lento proceso de construcción del sentimiento de nacionalidad en los países latinoamericanos; particularmente de ese sentimiento estructurado y promovido desde el Estado y difundido por medio de sus aparatos ideológicos y culturales, principalmente a través del sistema educativo. Así, en un período de casi sesenta años podemos advertir que en Nicaragua, entre 1871 y 1930, se impulsaron desde el Estado tres concepciones diferentes de lo que era la nación y la patria y tres interpretaciones distintas sobre el pasado del país, algunas de ellas incluso con matices contradictorios entre sí.

Estas variaciones en la construcción de un discurso nacional hegemónico sobre el pasado nicaragüense están íntimamente ligadas a los diferentes proyectos políticos, económicos y sociales de nación que se han impulsado en esos años, y al fracaso de cada uno de ellos. Estos fracasos que han imposibilitado que se consolide un proyecto de nación de largo plazo nos muestran que el Estado nicaragüense y la propia definición de Nicaragua como nación, son todavía muy frágiles, y que también se encuentran en proceso de construcción. Aún se encuentran inmersos en un proceso de búsqueda. Una búsqueda que se refleja en los textos escolares de Historia Nacional con que se promovió entre las generaciones de nicaragüenses de finales del Siglo XIX y primeras décadas del XX el sentimiento de pertenencia a la comunidad imaginada ${ }^{1}$ que es Nicaragua, es decir, los sentimientos de nacionalidad y patriotismo, y la fidelidad al propio Estado Nacional.

En este ensayo, a través del análisis de los textos escolares utilizado para la enseñanza de historia patria en esos años (1871-1930), exploraremos esos rumbos por los que ha transitado ese proceso de construcción de la nacionalidad nicaragüense patrocinada por el Estado y promovida en el sistema educativo. En el recorrido expondremos algunas de las contradicciones que se han presentado en esa búsqueda.

\section{Imagen de nación al final de los treinta años conservadores en el Siglo XIX}

En un texto fundamental para el estudio sobre el desarrollo de la idea de la nación nicaragüense la reconocida historiadora Frances Kinloch Tijerino sostiene que Tomás Ayón (18201887), autor del primer tratado sobre la Historia de Nicaragua escrito y publicado en el país, creó

1 Al referirnos a la nación, compartimos el planteamiento de Benedict Anderson, cuando define a la nación como "una comunidad política imaginada", y al nacionalismo como "un artefacto cultural de una clase particular”, Benedict Anderson, Comunidades Imaginadas. Reflexiones sobre el origen y la difusión del nacionalismo. (México: Fondo de Cultura Económica, 1993) 21-23. 
con su obra "un elemento fundamental del imaginario nacional: la idea de que los nicaragüenses constituían una colectividad con un pasado compartido desde los tiempos más remotos”. ${ }^{2}$ La observación de Kinloch Tijerino es acertada, pero se debe aclarar que ese importante "elemento del imaginario nacional” se mantuvo encerrado en muy pocas manos, pues no trascendió más allá de las élites y los pocos letrados que se contaban en Nicaragua en ese entonces. Por eso sería más preciso afirmar que el texto del reconocido diplomático y jurisconsulto constituye uno de los pilares fundamentales sobre los cuales se ha venido erigiendo un discurso hegemónico, oficial, sobe el pasado nicaragüense, orientado a la promoción de la nacionalidad nicaragüense. Otro de los pilares lo levantó pocos años después José Dolores Gámez (1851-1918), reconocido como el fundador de la historiografía nicaragüense. ${ }^{3}$ La difusión de un imaginario nacional -o lo que es lo mismo, la imagen de la nación y la patria-, extendida hasta en los pueblos pequeños o “secundarios” se dio años más tarde, cuando el gobierno de José Santos Zelaya impulsó entre 1893 y 1909 un proyecto de nación estructurado bajo los principios liberales. ${ }^{4}$ En esos años el número de escuelas primarias pasó de 193 a $643 .{ }^{5}$ Esta expansión del sistema educativo se interrumpió abruptamente cuando Zelaya fue expulsado del gobierno, y del país, por las presiones diplomáticas y las amenazas militares del gobierno estadounidense. ${ }^{6}$

Obviamente el esfuerzo liberal no fue el primer intento de difundir en todo el territorio nicaragüense una visión hegemónica de la nación. Años atrás los últimos gobiernos conservadores de los famosos treinta años, ${ }^{7}$ también intentaron de hacer lo propio. En sus famosas reflexiones sobre la Historia de Nicaragua, el poeta José Coronel Urtecho asegura que fue en este período, a de la década de 1870, cuando surgió entre las ideas de las élites conservadoras la necesidad de construir un relato histórico nacional que sirviera a la vez para la difusión de un sentimiento

2 Frances Kinloch Tijerino, Nicaragua: identidad y cultura politica. 1821-1858 (Managua, Nicaragua:

Banco Central de Nicaragua, 1999), 11.

$3 \quad$ Jorge Eduardo Arellano, Héroes sin fusil (Managua: Editorial Hispamer, 1998), 240.

4 Jilma Romero (coord.). Historia de Nicaragua. (Managua: Universidad Nacional Autónoma de Nicaragua, 2002), 184-185.

$5 \quad$ Isolda Rodríguez Rosales, La educación durante el liberalismo. Nicaragua: 1893-1909. (Managua: Editorial Banic, 1998), 199.

$6 \quad$ Un relato detallado de estos acontecimientos se puede consultar en Gregorio Selser, La restauración conservadora y la gesta de Benjamín Zeledón. Nicaragua-USA 1909-1916 (Managua, Aldilá Editor, 2001)

$7 \quad$ Después de la derrota de William Walker en 1856, en lo que se conoce en Nicaragua como la Guerra Nacional antifilibustera, a partir de 1857 hasta 1893 se sucedieron en el país siete gobiernos dirigidos por miembros del Partido Conservador. La historiografía nicaragüense denomina este período como "Los Treinta Años Conservadores”. 
nacional. ${ }^{8}$ Efectivamente, los primeros esfuerzos orientados hacia la construcción de un discurso histórico nacional fueron impulsados por los presidentes que gobernaron en la segunda mitad de los treinta años conservadores, especialmente Vicente Cuadra (1871-1875), Joaquín Zavala (1879-1883), y Evaristo Carazo (1887-1889). ${ }^{9}$ Estos presidentes, aunque se consideraban así mismos conservadores, en la práctica impulsaron políticas de corte liberal, al extremos que un sector de su partido llegó a acusarlos de no ser verdaderos ni legítimos conservadores. ${ }^{10}$ De tal manera que fue durante el período presidencial del conservador Vicente Cuadra (1871-1875) que se publicó el primer texto utilizado para la enseñanza de la historia de Nicaragua en los establecimientos de educación del país.

Esa obra titulada Notas geográficas y económicas sobre la república de Nicaragua, tiene una historia muy singular. En primer lugar no fue escrita por un escritor nicaragüense. Su autor fue Pablo Lévy, un ingeniero de origen francés que llegó a Nicaragua atraído por la posibilidad de la construcción de un canal interoceánico, proyecto que entonces se creía de próxima realización. Lévy anhelaba poner sus conocimientos científicos al servicio de esa obra. Según lo relata en el prólogo de su obra, una vez en Nicaragua consideró necesario escribir un texto que diera a conocer a Nicaragua en el exterior, ya que las obras referidas a Nicaragua publicadas hasta entonces, a pesar de sus indudables méritos, adolecían de muchas deficiencias. ${ }^{11}$ Con su escrito, el ingeniero francés se proponía además atraer inversionistas para el proyecto canalero y promover la inmigración europea a Nicaragua. Por consiguiente, tampoco estaba destinado, originalmente, para servir como texto escolar. Sin embargo, la obra dio un inesperado giro cuando las élites en el poder leyeron el manuscrito y se entusiasmaron de tal manera que

$8 \quad$ José Coronel Urtecho, Reflexiones sobre la historia de Nicaragua. De la colonia a la independencia (Managua, Nicaragua: Fundación Vida, 2001), 613.

$9 \quad$ El presidente Zavala suscribió un acuerdo con Tomás Ayón para que redactara un texto Historia de Nicaragua. Ayón proyectó escribir su obra en tres tomos, pero su repentina muerte impidió que la concluyera. 10 Según el historiador conservador, Emilio Alvarez Lejarza, estos presidentes "enderezaron al partido [conservador] hacia el liberalismo". Emilio Alvarez Lejarza, "El liberalismo en los treinta años", en Revista Conservadora del Pensamiento Centroamericano (Managua) 51 (Diciembre de 1964): 23. La historiadora mexicana María del Carmen Collado también ha señalado las inexactitudes de la tajante calificación de liberales y conservadores que normalmente se hace de los políticos de la época. Al poner de manifiesto muchas de las coincidencias en los planteamientos políticos y económicos de los dos partidos históricos de la Nicaragua del siglo XIX., sugiere que deben tomarse en consideración diversos matices. Ver María del Carmen Collado, "Liberales y conservadores de Nicaragua. ¿Falsos estereotipos?”, en Revista Secuencia (México) 11. (Mayo-Agosto de 1988), 65-76.

11 Pablo Lévy, Notas geográficas y económicas sobre la República de Nicaragua (Managua: Fondo de Promoción Cultural-Banco de América, 1976), xix y xx. 
propusieron fuera utilizado como texto para "instruir a la juventud", significando con ello que había que enseñar la historia y geografías nicaragüenses. El presidente Cuadra solicitó entonces al propio autor que la tradujera al español. ${ }^{12}$

En la primera sección de sus Notas geográficas y económicas, Lévy realizó una encomiable síntesis del pasado nicaragüense. Sin embargo, la imagen que el autor tenía del país, y por consiguiente la visión de la nación, se expresan mejor en los capítulos que dedica a la población, la etnología, y "geografía administrativa" nicaragüense. En esas secciones se refiere a las costumbres, los hábitos alimenticios, las expresiones religiosas, y a la composición étnica de la población nicaragüense. En sus observaciones el autor crítica "las imperfecciones" y todo lo que considera malo o negativo en el país. Utilizando un tono que recuerda Los viajes de Gulliver, Lévy critica desde el sabor del café que se prepara en Nicaragua hasta las manifestaciones en que la población expresaba su devoción católica, pasando por la forma en que los miembros de la élite gastaban su tiempo libre, el vestuario de los sectores más pobres de la población y la ineficiencia de la administración pública. La carga negativa que se observa en el texto de Lévy pareciera ofrecer nuevos argumentos, o corroborar los expresados, a favor de ese pensamiento ilustrado que hablaba de una supuesta degradación e inferioridad de la población americana y de su incapacidad para gobernarse a sí misma, ideas que tan "vehementemente" habían sido rechazado durante el período de emancipación colonial. ${ }^{13}$ La conclusión a la que llega Lévy es que Nicaragua no estaba "bien poblada”. Para él, principal problema del país era que la población era "esencialmente mestiza", hecho que consideraba sumamente grave porque "los mestizos son siempre seres inferiores, física y moralmente, a las razas puras que los han producido; [y] porque los mestizos actuales se sobrecruzan entre sí, y van, por consecuencia, inferiorizándose todos los días más y más”. Pero lo que Lévy consideraba todavía más “espantoso” era que la élite parecía no preocuparse de esa situación, y tampoco hacía nada por "remediarla”. Finalmente, el ingeniero galo proponía como solución urgente, si Nicaragua se quería "salvar", que se promoviera la inmigración de europeos, algo que debería de hacerse "por el bien de la humanidad". ${ }^{14}$

Los anteriores planteamientos han sido considerados casi como un exabrupto de Lévy,

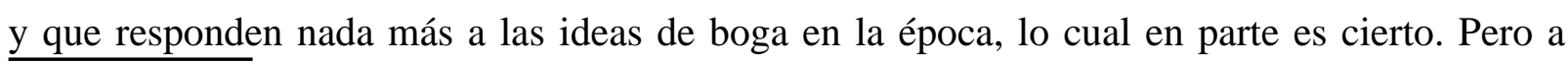
12 Lévy, xxii.

13 Frances Kinloch Tijerino, “La idea de nación en la Nicaragua de 1858”, en Memorias del IV Simposio Panamericano de Historia (México: Comisión de Historia - Instituto Panamericano de Geografía e Historia, 2001), 194.

14 Lévy, 193-194. 
nuestro juicio, por eso mismo esas ideas expresan la forma en que los miembros de las élites en el poder contemplaban y concebían al país. Sin lugar a dudas, ellos compartían en buena medida esa imagen de la nación. Debemos recordar que fueron representantes de estos grupos de poder los que solicitaron que el libro se tradujera al español y fuera utilizado como texto escolar. El único que criticó el texto de Lévy fue el cronista e historiador Jerónimo Pérez (1828-1884). Pero los señalamientos de Pérez tampoco estaban en contra de las concepciones racistas del autor, sino que más bien parecían orientadas a defender la gestión de los patriarcas conservadores y las manifestaciones religiosas de los nicaragüenses que Lévy tanto criticó. Lo que queremos señalar en estos breves párrafos es que el primer texto utilizado por jóvenes nicaragüenses para estudiar su historia y geografía, a finales del siglo XIX, ofrecía una imagen negativa de su propio país. Esa fue la imagen de la nación que interiorizaron los jóvenes que estudiaron este texto: una patria en la que coexistían dos grandes regiones, una "civilizada” y la otra no, y que la civilizada también dejaba mucho que desear pues tampoco marchaba bien. Aunque seguramente el universo estudiantil que aprendió de este texto fue muy pequeño, dado los altísimos niveles de analfabetismo en esos años.

En su texto Lévy también ofrece un valioso testimonio sobre los pobladores de la Costa Caribe nicaragüense, región que él llama "la parte no civilizada" de Nicaragua, sobre sus costumbres y su propia perspectiva respecto a la realidad del país, lo cual equivale a otra imagen o idea de la nación. Lo poco que dice Lévy de esta perspectiva, atribuida por el autor francés a la "incapacidad" de los indígenas de "comprender la igualdad republicana", nos sugiere que es una elaboración meditada, que los miembros de las comunidades indígenas construyeron a partir de su propia experiencia histórica. Lévy asegura que los indígenas, todos con los que pudo hablar, se esforzaban en demostrarle al viajero que el estilo o la forma de vivir de los no indígenas era la equivocada. En cambio, sostenían que a ellos, los indígenas, les asistía la razón al vivir de la forma en que vivían. Sin salir de su asombro, Lévy comenta que estos pobladores autóctonos representaban un fenómeno moral "talvez único en el mundo", "de ser salvajes con pleno conocimiento de causa y propósito deliberado". ${ }^{15}$ Hasta pareciera burlarse de esta visión indígena, hoy diríamos de la interpretación de su realidad histórica que hacían los pobladores caribeños, cuando afirma que "se imaginan" que en Nicaragua existen dos clases "una que oprime y otra que está oprimida”. Asegura que los indígenas estaban convencidos de

15 Lévy, 210. 
que si se "civilizaran", serían colocados en la segunda clase, y por ello se empeñaban en no salir de la situación en que se encontraban. "Su mayor temor, agrega, es que les hagan soldados y les manden después a hacerse matar por algún motivo desconocido de ellos”. ${ }^{16}$

Al consultar otras fuentes históricas podemos comprobar fácilmente que los planteamientos indígenas tenían su fundamento en la realidad que les había tocado vivir, y si no a ellos a otros indígenas, en sus interrelaciones con los habitantes de la costa del Pacífico, la "parte civilizada", Nicaragua y los nicaragüenses. El historiador y cronista Francisco Ortega Arancibia da cuenta de esas experiencias. En su relato del sitio al que los granadinos sometieron a los leoneses durante la guerra civil de 1844, cuenta que los sitiadores "tomaron indios de los que de Matagalpa llegaron, y los cargaron con unos sacos llenos de arena para que los pusieran en las calles, formando barricadas: los soldados [leoneses, sitiados] les hacían descargas de fusilería y los mataban; y aunque atemorizados lo demás no querían llevar más sacos de arena, los amenazaban los oficiales [sitiadores]; y los indios morían, hasta que por fin dispusieron hacerlas en la oscuridad de la noche”, ${ }^{17} \mathrm{El}$ cronista agrega que los granadinos después "repusieron" esas bajas con otros indios que habían llegado, entregándoles los fúsiles abandonados por los muertos y heridos. ${ }^{18}$

Es probable que Lévy desconociera esa realidad histórica. De lo contrario no se habría extrañado que los indígenas le tuvieran mucho "odio y a la vez mucho miedo" a los que mandaban y vivían en la parte “civilizada” del país.

Es muy significativo que cuando este texto fue reeditado más de un siglo después de su primera publicación, después de elogiar la labor realizada por el ingeniero francés, Jaime Incer Barquero, uno de los pocos científicos que se cuentan en Nicaragua, escribió en el prólogo que hay que "dispensarle sus especulaciones racistas (...) pues tales ideas eran muy aceptadas en el siglo pasado..." ${ }^{\prime 19}$ Probablemente le asista la razón al eminente científico cuando argumenta que esas eran ideas muy aceptadas en la época de Lévy, pero se equivoca al dejar pasar por alto el significado y las implicaciones políticas, económicas y sociales de dicho pensamiento, y sobre todo las reminiscencias que de esas ideas subsisten en nuestros días. Ideas que aunque ahora no se expresen de forma tan cruda no se puede negar que de una u otra forma han influido en los

16 Lévy, 249.

17 Francisco Ortega Arancibia, Cuarenta años de Historia de Nicaragua 1838-1878 (Managua: Fondo de Promoción Cultural del Banco de América, 1974), 61.

18 Ortega Arancibia, 61

19 Jaime Incer Barquero. "Un texto que hizo historia”, prólogo del texto de Pablo Lévy, Notas geográficas e Históricas de la República de Nicaragua. xi 
proyectos de nación, excluyentes, discriminadores y etnocéntricos, que se han impulsado en Nicaragua hasta ahora. $^{20}$

Pero eso explica, aunque no deja sorprender, que la parte que más llamó la atención a Incer Barquero fue el capítulo referido a temas etnológicos: costumbres, tradiciones y arquitectura nicaragüense. Nuestro científico asegura que a "todos aquellos que nacimos hace algún tiempo y gozamos de la solariega vida pueblerina y patriarcal de nuestros abuelos, la meditación de este capítulo nos traerá la evocación de aquellas épocas de rústica simplicidad e inocencia”. ${ }^{21}$ Asumiendo una perspectiva similar, también sería muy interesante saber qué evocaciones traería a los descendientes de “los caribes”, los actuales indígenas Mayagnas, entre los que convivió Lévy, si también llegara a ellos el texto y leyeran lo que el ingeniero francés escribió sobre sus antepasados.

Por lo que sabemos, como ya comentamos, a los pocos años el texto de Lévy se había agotado, y se hizo necesario redactar una nueva obra, ahora sí específicamente de Historia de Nicaragua, pensada explícitamente para ser utilizada en los centros escolares. Fue ante esta necesidad que surgieron los textos de Ayón y Gámez. ${ }^{22}$ Pero éstos resultaron muy densos, y se

$20 \mathrm{Al}$ analizar las variaciones en los datos referidos los indígenas en diferentes censos poblacionales practicados en Nicaragua, entre las últimas décadas del siglo XIX y las primeras del XX, Jeffrey Gould sostiene que el discurso de las élites dominantes acerca del mestizaje era "tan poderoso" [so powerful] que "cientos de miles de indios centroamericanos, como los Sutiavas, llegaron a avergonzarse de sus marcas indígenas, en la medida en que la palabra "indio" llegó a ser sinónimo de ignorante o salvaje. (So powerful was the dominant discourse that hundreds of thousands of Central American Indians, like Sutiavas, became "ashamed" of their ethnic markers, as the word Indian became synonym of "ignorant" or "savage”.) Jeffrey L. Gould. "iVana Ilusión! The Highlands Indian and the Miyth of Nicaragua Mestiza, 1880-1925", en Identity and Struggle at the Margins of the Nation-State. The laboring Peoples of Central America and the Hispanic Caribbean, edits. Aviva Chomsky y Aldo Lauria-Santiago (North Carolina, EUA: Duke University Press, 1998), 52. Sin embargo, hay que señalar que la discriminación hacia los indígenas no fue solo una cuestión de discurso. Fue una práctica agresiva, destructora de la identidad y de las formas de vida propia de las comunidades autóctonas de Nicaragua. Una práctica que se derivaba del proyecto de nación que en su momento impulsaron liberales y conservadores a finales del siglo XIX y principios del XX. Incluso, las políticas del gobierno revolucionario sandinista hacia las comunidades del Caribe, en la década de 1980, aunque llenas de "buena fe, entusiasmo" y de los ideales libertarios de la revolución, también estaban cargadas por “una buena dosis de prejuicios”. Ver Víctor S. Pozas. $L a$ revolución sandinista. 1979-1988 (Madrid: Editorial Revolución, 1988) y, especialmente, Jorge Jenkins Molieri. El desafio indígena en Nicaragua: el caso de los miskitos (Managua: Editorial Vanguardia, 1986)

21 En Lévy, xi

22 Años después de la publicación del texto de Lévy, que para entonces ya no se consideraba "apropiado" para las escuelas, el presidente Joaquín Zavala encargó a Tomás Ayón que escribiera una historia de Nicaragua. El proyecto quedó inconcluso por el fallecimiento del autor, quien en vida publicó solamente dos volúmenes de su obra. Poco después, en 1888, el presidente Carazo promovió un concurso nacional para la redacción de un tratado de Historia de Nicaragua que se utilizaría como texto escolar. La obra escrita por Gámez fue ganadora de ese 
hizo necesario redactar un nuevo texto, para explicar de forma sencilla las principales fechas y acontecimientos históricos a través de los cuales se ha ido forjando la nacionalidad nicaragüense. Pero lo que cristalizó completamente la redacción del nuevo texto de Historia de Nicaragua, y sobre todo su difusión, fue la llegada al poder de un nuevo proyecto político, y por consiguiente de una nueva visión de la nación.

\section{El catecismo de historia patria de los liberales}

Con la llegada al gobierno del liberal José Santos Zelaya, y al paso de las reformas modernizadoras que impulsó su gobierno desde 1893 -separación Estado-Iglesia, institución del matrimonio civil y el divorcio, educación laica, extensión del sistema educativo a todo el territorio nacional, entre otras-, la nueva idea de la nación en el poder se empeñó en acciones más decisivas para cohesionar y unificar políticamente los diversos conglomerados sociales y étnicos existentes en el país. De ahí que una de sus principales prioridades haya sido tratar de imponer en la Costa Caribe los mismos patrones educativos concebidos desde y para la costa del Pacífico. Su objetivo era asimilar a la población indígena de la zona y convertirlos en nicaragüenses. Esta política enfrentó una fuerte resistencia de parte de "los costeños", ${ }^{23}$ pero de todas maneras la educación asumió el papel de "máquina cultural” que "difundió contenidos nacionalistas que reprimieron las particularidades culturales de origen, imponiendo un modelo de integración ciego a las diferencias”, en palabras de la argentina Beatriz Sarlo. ${ }^{24}$ Las herramientas fundamentales utilizadas en esas máquinas culturales para construir un pensamiento hegemónico sobre la patria y la nación fueron los “catecismos” de Historia Patria y de Historia de Centroamérica, escritos por Gámez,

La primera edición del Catecismo de Historia Patria, se publicó en 1889, durante el gobierno del conservador Evaristo Carazo (1887-1899) y es un resumen en extremo esquemático de la principal obra de Gámez, su Historia de Nicaragua. Desde los tiempos prehistóricos hasta 1860, en sus relaciones con España, México y Centro-América. Esta breve obra fue el primer texto de carácter oficial utilizado de manera obligatoria en las escuelas públicas para el estudio de la asignatura de Historia Patria, establecida por primera vez en el país por el gobierno

certamen.

23 Isolda Rodríguez Rosales, La educación durante el liberalismo. Nicaragua 1893-1908 (Managua:

Editorial Banic, 1998), 182-190.

24 Beatriz Sarlo, La máquina cultural. Maestras, traductores y vanguardistas (Buenos Aires: Ariel, 1998),

28. 
liberal de Zelaya como asignatura de estudio en los programas de las escuelas primarias. ${ }^{25}$ En la introducción, Gámez desarrolla una explicación breve y bastante simple para delimitar la idea de nación y circunscribirla sólo a Nicaragua, derivándola del concepto que incluía a toda Centroamérica. Estructura su argumento a partir de su concepción de la Historia y de la forma en que ésta puede dividirse o periodizarse. Así, según él, la historia general corresponde a toda la región del istmo hasta 1838, año en que se fraccionó la República Federal de Centroamérica. Después de esa fecha, cada unos de los segmentos en que se dividió Centroamérica tiene una historia particular que lleva el nombre de la localidad respectiva: historia de Guatemala, de El Salvador, de Nicaragua, etcétera. Por consiguiente, hasta 1838 la Historia Patria incluye a la de toda Centroamérica; pero después de ese año la Historia Patria se limita o se reduce a la Historia de Nicaragua, que anteriormente podría ser considerada como historia particular. ${ }^{26}$ En correspondencia con esto, en la primera sección del libro se referirá a Centroamérica como el país o la nación. Así, cuando se pregunta cuál era el idioma más usado en el país antes de la llegada de los españoles, enumera las lenguas que predominaban mayoritariamente en el territorio de lo que posteriormente sería cada uno de los estados centroamericanos. ${ }^{27}$ En la última sección del texto, que da el mismo título que dio a otra de sus grandes obras (Historia Moderna de Nicaragua), se referirá exclusivamente a los acontecimientos ocurridos en el territorio nicaragüense después de la ruptura del pacto federal. Así, la patria se ha reducido entonces a Nicaragua.

En el Catecismo de Historia de Centroamérica, para el curso del mismo nombre establecido en la enseñanza intermedia o bachillerato, Gámez define a Centroamérica como "la antigua patria de los nicaragüenses”, dando a entender que esa ya no es la patria, pues como dejó sentado en su otro Catecismo, la patria ahora se delimitaba a Nicaragua. No obstante recalca que es importante conocer la historia del istmo porque pese a la ruptura de la República Federal de Centroamérica, que él denomina "fraccionamiento político" de la patria, no se rompieron "los lazos de familia de las cinco repúblicas aún hermanas, que siguen unidas por el origen, el sistema de gobierno, sus comunes intereses, por sus aspiraciones y creencias”. ${ }^{28}$ Gámez recurre así elementos considerados constitutivos de la nación: el origen común, es decir, un mismo

25 Carlos Molina Argüello, La enseñanza de la Historia en Nicaragua. (México: Comisión de Historia del Instituto Panamericano de Geografía e Historia, 1953), 59.

26 José Dolores Gámez, Catecismo de Historia Patria (s/d. 1894), 10-12.

27 Gámez, 27.

28 José Dolores Gámez, Catecismo de Historia de Centroamérica (Managua: Tipografía Nacional, 1900),

4. 
pasado, las tradiciones y creencias, la comunidad de intereses y hasta cierto destino común (las aspiraciones). No obstante, aunque no tuviera clara esas diferencias teóricas que en realidad se desarrollaron posteriormente, los términos que utiliza el historiador nicaragüense le funcionan para abordar una realidad histórica: Centroamérica como unidad geográfica, histórica y política; una entidad que se disgregó en fragmentos que a partir de 1838 tomaron cinco derroteros distintos aunque no completamente diferentes. Así reafirma nuevamente su idea de que la historia de Centroamérica comprende desde el período prehispánico hasta "el último simulacro de gobierno federal que sostenía la gloriosa espada del general Morazán”; y que esa historia nacional después se fragmentó en cinco secciones que con los años fueron denominadas también historias nacionales, correspondiente a la historia de cada uno de los países en que se fraccionó la República Federal de Centroamérica. ${ }^{29}$

Lo que podemos deducir de las ideas expresadas por Gámez en ambos "catecismos” es que a través de ellos se transmitió a toda una generación de nicaragüenses un doble sentimiento de nacionalidad. Lo jóvenes de la época aprendieron que su patria era Nicaragua; y su nación, o país al que pertenecían, era Centroamérica. Esta concepción de la nación de Gámez está en total correspondencia con la forma en que se define a Nicaragua en la Constitución Política promulgada en los primeros años del gobierno de Zelaya. El artículo primero del Título I de la famosa "Libérrima" de 1893 reza que Nicaragua es "una sección disgregada de la República de Centroamérica"; aunque posteriormente también especifica, en el artículo dos, que "Nicaragua es una nación libre y soberana”. ${ }^{30}$ Recordemos que Gámez como Zelaya fueron devotos del ideal unionista centroamericano, lo cual los habría llevado incluso a combatir al lado del presidente guatemalteco Justo Rufino Barrios, que en 1885 hizo el último intento por reconstituir a la fuerza la unidad centroamericana. Este doble sentimiento de nacionalidad también puede ser visto como una resonancia de lo que Marta Elena Casaús Arzú y Teresa García Giráldez llaman la "cohabitación” e interactuación de dos lealtades que se dio en los posteriores inmediatos a la independencia de la Capitanía General de Guatemala: "la lealtad a la patria centroamericana, a una entidad grande" y la lealtad "a la patria chica, entendida como el lugar de origen”. ${ }^{31}$ En esas

29 Gámez, Catecismo de Historia de Centroamérica, 4

30 “Constitución de 1893”, Biblioteca Virtual Miguel de Cervantes.

http://www.cervantesvirtual.com/servlet/SirveObras/12837219748926051876435/p0000001.htm

(Fecha de acceso: 22 de marzo del 2008)

31 Marta Elena Casaús Arzú y Teresa García Giráldez, Las redes intelectuales centroamericanas: un siglo de imaginarios nacionales 1820-1920 (Guatemala: F\&G Editores, 2005), 52. 
dos aguas navegaban los conceptos de nación y patria que los liberales nicaragüenses de finales del XIX y principios del XX querían inculcar en las generaciones más jóvenes de nicaragüenses durante esos años.

\section{La patria confesional de la restauración conservadora}

El proyecto de nación de Zelaya fue interrumpido abruptamente cuando el caudillo liberal renunció al poder por las amenazas contenidas en la tristemente célebre nota firmada por el Secretario del Departamento de Estado de Estados Unidos, Philander Knox. Pocos años después de su partida, en 1913, de conformidad con la voluntad y los planes estadounidenses, y bajo la atenta vigilancia de las tropas EUA que para entonces ya ocupaban el país, fue electo presidente el político conservador Adolfo Díaz. Así iniciaba un nuevo proyecto político y una nueva etapa de la historia nacional que la historiografía nicaragüense ha bautizado como "la restauración conservadora”.

El nuevo proyecto de nación encabezado por Díaz y el Partido Conservador era muy singular: se pretendía el establecimiento de un protectorado estadounidense en Nicaragua, y para ello propusieron lo que el investigador argentino-mexicano Gregorio Selser denominó como "la nicaraguanización” de la Enmienda Platt. Con ese objetivo redactaron una versión nicaragüense de las disposiciones impuestas por el gobierno estadounidense al pueblo cubano en 1901. Al decir de Selser, lo que redactaron los políticos nicaragüenses en realidad no fue más que una calca del documento que la Convención Constituyente cubana se vio obligada aprobar e integrar como un apéndice en la Constitución cubana. Lo único que variaba, afirma Selser, era que se escribió Nicaragua en lugar de Cuba. ${ }^{32}$

Los conservadores tenían diecisiete años de acariciar el anhelo de retornar al gobierno y reconstruir el mítico status quo de los treinta años en los que gobernó su partido a finales del siglo XIX. Obviamente no podían dar marcha atrás en la historia ni deshacer las transformaciones experimentadas en Nicaragua después de diecisiete años de gobierno liberal zelayista. Aunque lo hubieran pretendido no hubieran podido restablecer el concordato vigente en Nicaragua desde 1861 hasta 1893, cuando Zelaya llegó al gobierno; ni conculcar el derecho al voto que ya se había ampliado a todos los varones mayores de 21 años que supieran leer y escribir, para delimitarlo nuevamente a las personas que tuvieran determinado capital, como lo establecía la Constitución de 1858. Sin embargo, hicieron lo que pudieron, o mejor dicho, lo que las nuevas

$32 \quad$ Selser, 389. 
condiciones les permitieron. En este sentido se empeñaron en difundir los valores ideológicos y morales que consideraban se habían perdido o estaban muy debilitados a consecuencia de la revolución liberal. Y es aquí donde la educación, y la enseñanza de la historia en particular, jugará nuevamente un papel decisivo. Había pues que difundir la nueva concepción de patria y nación que abrigada el nuevo grupo en el poder. Así, una de las primeras decisiones de los gobernantes conservadores fue elaborar una nueva Constitución Política que se promulgó en diciembre de 1911. Esta nueva constitución conservadora restablecía algunos de los privilegios con que contaba la Iglesia antes que Zelaya llegara al gobierno. Específicamente se omitía el carácter "laico" de la educación pública, y se reintroducía como obligatoria la enseñanza de la religión católica en las escuelas del país. En definitiva, era una constitución que retomaba los principios sociales que inspiraron la carta magna de 1858, promulgada al inicio de los míticos treinta años de gobierno conservador, según lo expresado por Carlos Cuadra Pasos, uno de los principales ideólogos del Partido Conservador e integrante del equipo que redactó la nueva Ley Fundamental de Nicaragua. ${ }^{33}$ De acuerdo con ese precepto constitucional se promulgó un nuevo reglamento de las escuelas primarias que establecía que uno de los principales objetivos de éstas era proveer a los niños y jóvenes nicaragüenses una educación “moral y religiosa”. Posteriormente el ministro del ramo emitió una comunicación en que dejaba claro que las clases de moral y religión tenían carácter obligatorio en todos los centros educativos públicos. La disposición del ministro también estipulaba que los padres de familias que no quisieran que sus hijos recibieran esos cursos tenían que explicarlo y solicitarlo por escrito. ${ }^{34}$ No se cuenta con datos sobre cuántos padres de familia habrían solicitado que exoneraran a sus hijos de la obligación de recibir religión en los centros escolares, pero es fácil suponer que no habrían sido muchos, y lo más probable es que ninguno lo haya hecho. Si acaso algunos lo solicitaron, debieron ser casos muy extraordinarios. Tomando en consideración los altísimos niveles de analfabetismo que imperaban entonces, no estaría muy lejos de la realidad pensar que muchos de estos padres de familias, especialmente de familias pobres o habitantes de las zonas rurales, ni siquiera se hayan enterado de que contaban con esa posibilidad.

La decisión de cargar la educación con un fuerte contenido religioso comenzó a llevarse a la práctica desde el mismo momento en que el gobierno otorgó un papel protagónico

33 Citado en Isolda Rodríguez Rosales, Historia de la Educación en Nicaragua. Restauración Conservadora 1910-1930 (Managua: Editorial Hispamer, 2005), 42.

34 Ibid., 77. 
a algunas órdenes religiosas, especialmente la de los Hermanos Cristianos de La Salle, que tenía presencia en Nicaragua desde 1903. Estos se hicieron cargo de aspectos medulares de la educación, incluyendo la redacción de los textos de historia. De hecho, la creación de la primera Escuela Normal de Maestros estuvo a cargo de los religiosos jesuitas. Rodríguez Rosales hace hincapié en que con la nueva orientación que se le daba a la educación el grupo conservador en el poder se proponía restituir el carácter humanista que la enseñanza habría perdido durante los diecisiete años liberales, debido al énfasis que el gobierno de Zelaya habría puesto a los aspectos utilitarios, técnicos y cienficistas. ${ }^{35}$ A nuestro juicio, la intención iba mucho más allá que promover determinados valores morales, religiosos o éticos abstractos. Es evidente que la decisión de entregar la educación a instituciones religiosas buscaba principalmente extender nuevamente la influencia de la Iglesia Católica otorgándole una nueva vía de transmisión de su mensaje alienante, adormecedor de las consciencias de una población a la que se le habían despertado muchas inquietudes sociales durante los años liberales. La Iglesia Católica recuperaba así su papel de adoctrinadora ideológica tal como lo había hecho durante la conquista y el período colonial para garantizar el sometimiento y la docilidad de los indígenas, ahora orientado hacia los descendientes de estos. Así lo reconoce implícitamente el Ministro de Instrucción Pública durante el gobierno de Diego Manuel Chamorro en 1921, al expresar su concepción de las funciones de las escuelas como lugares de "sumisión”, actitud consideraba uno de las principales virtudes legadas sus antepasados indígenas, y en donde la impetuosidad debería transformarse en “disciplina respetuosa y consciente". "Se esperaba [que] por medio de la educación se transformara al individuo, transformación que se lograría mediante la inculcación de la obediencia y la disciplina", afirma la historiadora nicaragüense. ${ }^{36}$ La obediencia y el respeto que se pretendía inculcar sienta sus raíces en determinada concepción del mundo que considera que todo ha sido creado por un ser superior que también ha determinado que las cosas sean tal a como son, y que los acontecimientos responden a su voluntad. Es la concepción que aprendieron los niños nicaragüenses que utilizaron los nuevos textos de historia elaborados por los religiosos, textos que también exaltarán el papel “civilizador” que se atribuye a la conquista europea.

Dos ejemplos de estos nuevos textos en los que se mezcla la concepción de la historia con la religión católica y en los que la idea de nación y patria está subordinada a esos preceptos religiosos son los escritos por los sacerdotes Bernardo Portas y Félix Pereira y Castellón. El $35 \quad$ Ibid., 58 .

36 Ibid., 58. 
texto de Portas, jesuita que había arribado a Nicaragua procedente de México apenas unos años antes, se titula Compendio de Historia de Nicaragua. Fue escrito y publicado en 1918, en momentos en que el país se encontraba ocupado por tropas de Estados Unidos. Está dedicado "al excelentísimo señor general Emiliano Chamorro, presidente de la república”, dirigente del Partido Conservador y uno de los principales aliados del gobierno estadounidense y promotor de la ocupación militar estadounidense. A nuestro juicio, la dedicatoria a ese personaje revela cuando menos una clara identificación del religioso con el proyecto político conservador, y muy probablemente una muestra de agradecimiento o admiración hacia el político que firmó con el gobierno estadounidense el oneroso tratado por el cual se cedía a perpetuidad el derecho de construir un canal interoceánico en territorio nicaragüense y se arrendaban por noventa y nueve años varias islas del Caribe nicaragüense y las costas del golfo de Fonseca, en el Pacífico, para que la fuerzas armadas estadounidense establecieran una base naval en cualquier momento que lo consideraran necesario. A cambio de esas concesiones, el gobierno de EUA se comprometía entregar al de Nicaragua tres millones de dólares. ${ }^{37}$

Pero más allá de las anteriores valoraciones éticas y políticas, el libro de Portas es uno de los primeros textos escolares de Historia Nacional que al menos en su aspecto formal parece escrito con mayor rigor académico. Portas compiló diversas fuentes, de las que ofrece algunos datos bibliográficos al final de cada uno de los capítulos de su libro, ${ }^{38}$ aunque curiosamente solo cuando cita a Gámez añade una singular nota al pie en la que advierte: "véase todo lo que dice este autor acerca de liberales y conservadores y nótese que él siempre fue liberal”. 39

En el recuento del pasado nicaragüense que hace Portas en su texto tiene un enorme peso la valoración que ofrece del proceso de conquista y del período colonial. El autor incluye

37 Dicho acuerdo, conocido como "Tratado Chamorro-Bryan", fue firmado el 5 de agosto de 1914 por el general conservador mientras fungía como representante de Nicaragua en Washington y el Secretario de Estado estadounidense William Jennings Bryan. Véase Selser, 408-419, quien reproduce el texto integro de este acuerdo, abrogado el 14 de julio de 1970 .

$38 \quad$ Entre los textos citados por el jesuita encontramos obras consideradas clásicas para el estudio de la historiografía centroamericana, como las Memorias para la historia del antiguo reino de Guatemala de Francisco García Peláez; el Compendio de Historia de la ciudad de Guatemala, de Domingo Juarros; el primer volumen de la Historia de América Central de José Milla, además las obras de Alejandro Marure, Lorenzo Montúfar, Manuel Montúfar, y las Memorias de Manuel José Arce y Miguel García Granados. Portas también consultó las historias de Centroamérica escritas por el estadounidense Hubert Bancroft, y las de los autores nicaragüenses Ayón, Jerónimo Pérez y José Dolores Gámez

39 Bernardo Portas S. J., Compendio de la Historia de Nicaragua (Managua: Tipografía y

Encuadernaciones Nacionales, 1918) 120. 
un capítulo titulado "Juicio general de la conquista española”, y en él es evidente la intención de borrar la imagen que introducían los catecismos patrios de Gámez, en los que se señalaba sin ambages las atrocidades que los europeos cometieron contra las poblaciones autóctonas. Portas en cambio ofrece explicaciones que justificarían el proceder de los conquistadores, pero de forma más contundentes de la que lo hizo Ayón en su Historia de Nicaragua, o como lo hiciera el historiador mexicano Lucas Alamán. ${ }^{40}$ Esta intención concuerda plenamente con la visión conservadora del pasado colonial como período de paz y etapa civilizatoria que años más tarde el poeta José Coronel Urtecho articulará en un discurso mucho mejor elaborado. ${ }^{41}$

Al resumir su juicio Portas insiste en que el sistema colonial español "a pesar de sus defectos, fue el mejor de aquellos tiempos”. En una referencia implícita y contradiciendo la interpretación que hace Gámez de este período, el religioso enfatiza que "[La] colonia española no fue aquella época de oscurantismo y miseria continua que algunos tanto ponderan”, pues a la par de los tributos y las injusticias "hubo también alegrías y bienestar y vida civilizada, no al modo nuestro moderno, sino al modo de la Europa de aquellos siglos”. ${ }^{42}$ Pero el legado que más encomia resulta fácil de imaginar: es el de la fe y la religión católica, lo que justificaría de sobra cualquier "innegable abuso" contra los habitantes originales de estas tierras. "España, a trueque del oro que sacó de nuestras tierras nos dio en hombres y en instituciones cuanto tenía y no puede exigírsele más. Nos inculcó sobre todo su profunda fe, esa fe única verdadera, para los que saben considerar las cosas como en realidad son, es el bien más preciado que puede poseerse sobre la tierra”, ${ }^{43}$ se expresa en el texto.

Posteriormente, al final de su Compendio, Portas también hace una idílica recordación de los treinta años de gobierno conservadores en el Siglo XIX. Así relata que después de la guerra nacional, la historia nicaragüense dio un giro completamente diferente porque apareció "un grupo de hombres escogidos que se van sucediendo en el gobierno del país, lo encausan

$40 \quad$ En sus Disertaciones como en Historia de México, Alamán se empeño en destacar las instituciones coloniales que consideraba habían sido "benéficas" para México. En su Historia, resulta "el apologista más apasionado” del conquistador Hernán Cortés. Ver María del Carmen Velázquez. "Lucas Alamán, Historiador de México”, en Silvio Zavala (coord). Estudios de Historiografia Americana. (México: El Colegio de México, 1948), 398.

41 En sus Reflexiones, ya citadas, Coronel Urtecho idealiza el período colonial nicaragüense, presentándolo como un modelo de sociedad ideal en el que toda la población gozaba de paz De esta manera, Coronel Urtecho atribuyó todos los males que ha sufrido Nicaragua (dictaduras, guerras civiles y otros conflictos sociales) a la ruptura de ese orden colonial, provocado por la independencia de Centroamérica.

42 Portas, 80.

43 Ibid., 80. 
con mano firme por el camino del progreso, venciendo con facilidad los conatos de trastornar el orden (...) las escuelas se multiplican, el comercio y la industria comienza a florecer...". ${ }^{44}$ Esa visión del jesuita ciertamente tiene un vínculo con la realidad, pero no es toda la realidad de ese período, y hay muchos detalles importantes que se cuida de mencionar. Por ejemplo, el carácter elitista y oligárquico de esos gobiernos, dado que la Constitución aprobada en 1858 redujo drásticamente las posibilidades de acceder a cargos públicos y a la primera magistratura de la nación a las personas que no tuvieran determinado capital. Esto explica que solo un grupo muy reducido, en algunos casos unido por vínculos familiares o relaciones de amistad, se traspasaran las riendas del gobierno sin mayores repercusiones políticas y sociales, lo que al final de cuentas representaba un significativo retroceso en la democratización del ejercicio público propuesto los liberales. Portas no ofrece ninguna pista sobre estos elementos que también son importantes para tener en cuenta en el relato histórico de la nación.

Es curioso que en el libro de Portas, a diferencia de lo que se espera de otros textos escolares de Historia Patria o Nacional, no resulta muy evidente el propósito de promover el patriotismo o el sentimiento de nacionalidad. Este hecho se hace más notable al leer las lecciones y fragmentos dedicados a los principales acontecimientos históricos, pues no se presta a enaltecer a esas figuras del pasado que estarían llamadas a ser ejemplo y objeto de respeto y veneración de parte de las nuevas generaciones de ciudadanos. Dos razones podrían explicar esto. Una: que el autor no era originario de Nicaragua ${ }^{45}$ y además tenía pocos años de residir en el país. Por consiguiente, no podía contribuir a transmitir un sentimiento patriótico o afecto nacional que él mismo no experimentaba. La otra, que la intención del texto pareciera ser más bien la de corregir los valores del catecismo cívico y patriótico que se enseñaba en tiempos de los liberales, y en este sentido la prioridad era promover los valores religiosos. De ahí que el mayor peso de su contenido sea la defensa de la "evangelización” durante la conquista y la colonia, y la construcción de una nueva imagen de este período histórico, una imagen más amable, cuyos rasgos más destacados están en la supuesta la tarea de "civilizar” a los habitantes originales del territorio que hoy es Nicaragua. Esa es la idea de la nación que ofrece, una idea que si bien no es explícita, se estructura en la nostalgia del pasado coloquial y del período de los treinta años de

$44 \quad$ Ibid., 147.

45 Según algunas fuentes, Portas era oriundo de España. En cambio, Jorge Eduardo Arellano, en su Diccionario de Autores Nicaragüenses. Tomo II (Managua: Biblioteca Nacional Rubén Darío, 1994), sostiene que era de nacionalidad mexicana. 
gobierno conservador representados de forma idílica.

El texto escrito por Pereira y Castellón, que también consideramos representativo de este período, se titula Cartilla de Historia de Nicaragua para uso de los hijos de los obreros, y es de suponer que se utilizó principalmente en las escuelas públicas, que eran las únicas donde podían asistir "los hijos de los obreros". Publicado en 1928, al igual que en el texto de Portas predomina en sus páginas el sentimiento religioso católico, al extremo que a veces pareciera ser más una historia religiosa, o recuento de hechos religiosos o de la Iglesia Católica, que una historia del país. Pero a diferencia del Compendio, el autor de la Cartilla, que sí es nacional de Nicaragua, declara inequívocamente su intención de cultivar y promover en los alumnos sentimientos patrióticos y de identidad nacionalista. Pereira manifiesta su intención patriótica del texto desde el mismo prólogo, al introducir un verso latino que él mismo se encarga de traducir. La frase dice: “aprende, oh niño, a conocer la patria por tu historia”. Seguido del verso, el autor se identifica como nicaragüense y expresa su amor a la patria que es la misma del público al que está destinado el texto, estableciendo así una empatía con sus posibles lectores. La obra, dice el autor, también es una muestra de cariño a los hijos del pueblo; y espera con ella despertar en sus corazones "el nobilísimo sentimiento del patriotismo, que no es otra cosa sino el anhelo constante de honrar con tu conducta moral y cristiana el pedazo de tierra que te vio nacer" ${ }^{46}$ En esta concepción del patriotismo Pereira no solo hace una paráfrasis de uno de los diez mandamientos bíblicos, sino que la concepción misma está imbuida de esa fe: Ser un buen patriota sería entonces amar y honrar el suelo donde uno nació, de la misma manera que para ser buen cristiano hay que "honrar a padre y madre”, según lo establecen las míticas tablas que de acuerdo con la leyenda bíblica habría recibido Moisés. Pereira también recomienda a los infantes que, una vez que sean mayores, lean las obras primarias de la historia nicaragüense como las de Ayón o Lévy “o de cualquier otro autor". Pereira también identifica el amor a la patria y ser buen patriota con el mantenimiento de la unidad de la nación. Así, al referirse a las disputas entre los dos principales centros urbanos de Nicaragua -León y Granada- tras la independencia centroamericana, aprovecha para reiterar su visión del patriotismo y el nacionalismo. Para este religioso, un verdadero patriota es quien contribuye a conservar la unidad de la nación. "El nicaragüense localista será si se quiere un eminente sabio, un digno sacerdote, un probo

$46 \quad$ Félix Pereira y Castellón, Cartilla de la Historia de Nicaragua para uso de los niños hijos de obreros. (León, Nicaragua: s/i, 1928, 2da Edición). Prólogo. 
magistrado, pero nunca un legitimo patriota”, sentencia. ${ }^{47}$

Estas cortas citas que hemos extraído sintetizan el sentimiento de nacionalidad que el texto de Pereira promovía en las clases populares; un sentimiento en el que el amor a la patria marcha indisolublemente vinculado a la fe católica y a los principios morales cristianos; lo que hace que el libro resulte una curiosa mezcla de catecismo para el adoctrinamiento religioso y los principios ideológicos patrióticos que Gámez quería transmitir con su catecismo patrio.

Los textos de Portas y Pereira sirvieron de inspiración a otros hermanos lasallistas, incluso a historiadores laicos vinculados con los centros de enseñanza regentados por esa y otras órdenes religiosas. Así surgieron los textos de historias para estudiantes de tercero y cuarto grados de primaria, publicados años más tarde, a inicios de la década de 1940, durante un nuevo contexto político y cuando ya había quedado atrás la restauración conservadora. En los primeros años de la dictadura de los Somoza, el fundador de la dinastía, Anastasio Somoza García, aunque se proclamaba liberal y admirador de la obra de Zelaya, no tuvo muchos problemas para ceder a la demanda de los conservadores de que en las escuelas públicas se siguieran inculcando los “valores cristianos”. ${ }^{48}$ Más aún si eso le permitía continuar en el poder. Cuando llegó el turno para que los hijos de Somoza García gobernaran el país, la situación fue similar. Si bien a finales de la década de 1960 se publicaron libros bajo el influjo del proyecto integrador centroamericano, que pretendieron unificar la educación en todo el istmo, también se siguieron utilizando los textos que promovían una idea de nación y sentimientos patrióticos vinculados a la religión católica. El extremo de esto lo representa el texto Lecciones de geografía e historia de segundo grado, elaborado por el Colegio Calazans para sus alumnos. Por medio de ese libro se enseñaba a los pequeños que "Nicaragua es nuestra patria. Los montes y volcanes, los lagos y los ríos, los bosques y prados, los llanos y valles, nuestro cielo y nuestro mar... todo es Nicaragua”, pero también se les pedía recordar que "Nicaragua es también, y principalmente, nuestra Santa Religión, nuestra lengua, las cenizas de nuestros mayores y la memoria de nuestro glorioso pasado". 49

Textos como el del Calazans, y otros similares, aunque fueron elaborados principalmente para los alumnos de escuelas religiosas también eran utilizados por docentes y alumnos de los

$47 \quad$ Ibid., 161

48 Knut Walter. El régimen de Anastasio Somoza 1936-1956. (Managua: Instituto de Historia de Nicaragua y Centroamérica-Universidad Centroamericana, 2004), 286.

49 Colegio Calazans. Lecciones de geografia e historia de segundo grado. (Sin datos), p. 24-25 
colegios estatales. Su distribución en diversas librerías del país y la falta de otras opciones facilitaban esa situación, que prevaleció hasta finales de la década de 1970, cuando el país experimentó un nuevo cambio político radical, que en su momento también promovió la redacción y publicación de nuevos, diferentes textos para la enseñanza del pasado nicaragüense.

\section{Conclusión}

Este breve análisis de los discursos y las representaciones sobre la nación que se transmitieron en los textos utilizados para la enseñanza de la historia de Nicaragua entre 1873 y 1930, nos lleva a varias conclusiones. En primer lugar, que la enseñanza de la historia del país ha estado signada por constantes y abruptas rupturas que se traducen en cambios radicales en los contenidos de los textos, pero sobre todo en la interpretación de los hechos históricos. Esto obviamente también ha tenido claras implicaciones en la concepción de patria y nación con que crecieron las generaciones de nicaragüenses en esos años. Así tenemos que entre 1893 y 1909 se impulsó desde el Estado una idea de nación en la que tenían gran preeminencia los principios liberales, pero que también mostraba una vocación centroamericanista, pues además de entender la nación como el territorio donde se ha nacido, en este caso Nicaragua, también alentaba la idea de que el país era un fragmento de una patria más grande, Centroamérica. En otras palabras, se promovía fuertemente el espíritu de unión centroamericana, labor a la que el propio presidente Zelaya se abocó, aunque con resultados infructuosos.

Después de la caída de Zelaya en 1909, cuando se dio uno de los primeros cambios radicales que sufrirá la enseñanza de la historia nicaragüense en el Siglo XX; cuando el grupo que tomó el poder impulsó una nueva concepción de la nación. Este grupo retomó la vocación tradicionalista y clerical de los conservadores de finales del siglo XIX, impregnaron el contenido de los textos de historia con su propia fe confesional católica, de manera que en su concepto de patria se evidencia una fusión y confusión entre patriotismo, sentimiento de nacionalidad y fe religiosa católica. Esta visión fue la hegemónica durante el período que la historiografía nicaragüense denomina como la "restauración conservadora" (1911-1930), y no desapareció aunque los representantes de la dictadura que se estableció a partir de 1936 se declararon seguidores de la corriente política opuesta; de modo que su influencia se sentirá con fuerza hasta finales de los años setentas.

Estas continuas variantes en la promoción de un discurso oficial sobre el pasado nicaragüense sin lugar a dudas también son una manifestación de los diversos tropiezos que 
se ha enfrentado a lo largo del proceso de construcción del Estado nicaragüense, el cual aún es nuestros días se manifiesta muy frágil. Y esa fragilidad el Estado nicaragüense es a su vez manifestación de una realidad mucho más compleja: el hecho de que hasta ahora no se ha logrado consolidar ningún proyecto de nación. En este sentido Nicaragua ha estado y aún está en constante búsqueda, una búsqueda en la que ha sufrido dolorosos tropezones y sangrientas caídas. Por ello consideramos que Nicaragua tiene un carácter de nación abortada, o en continuo proceso de formación. Los diferentes proyectos políticos que podrían constituirse como tales han sido ahogados en sangre; siendo el más reciente el proyecto impulsado el sandinismo en la década de 1980. Y las perspectivas tampoco son halagadoras, pues el último cambio de signo político en las estructuras de poder en Nicaragua está ensombrecido por fuertes manifestaciones de inestabilidad, y es muy probable que la generación de nicaragüenses, que apenas ahora empieza a crecer, cuando inicie su vida escolar y aprenda en sus textos de Estudios Sociales una interpretación de su pasado y adquiera una nueva idea de la nación diferente a la que se ha difundido en los últimos diecisiete años.

\section{Bibliografía}

Alvarez Lejarza, Emilio. “El liberalismo en los treinta años”, Revista Conservadora del Pensamiento Centroamericano. (Managua) 51 (Diciembre de 1964), 23-33.

Anderson, Benedict. Comunidades imaginadas. Reflexiones sobre el origen y la difusión del nacionalismo. México: Fondo de Cultura Económica, 1993.

Arellano, Jorge Eduardo. Héroes sin fusil. Managua: Editorial Hispamer, 1998.

Casaús Arzú, Marta Elena y Teresa García Giráldez. Las redes intelectuales centroamericanas: un siglo de imaginarios nacionales 1820-1920. Guatemala: F\&G Editores, 2005.

Colegio Calazans. Lecciones de geografia e historia de segundo grado. Sin datos.

Collado, María del Carmen. “Liberales y conservadores de Nicaragua. ¿Falsos estereotipos?”, Secuencia (México) 11 (Mayo-Agosto de 1988), 65-76. 
“Constitución de 1893”, Biblioteca Virtual Miguel de Cervantes.

http://www.cervantesvirtual.com/servlet/SirveObras/12837219748926051876435/p0000001. htm

(Fecha de acceso: 22 de Marzo del 2008)

Coronel Urtecho, José. Reflexiones sobre la historia de Nicaragua. De la colonia a la independencia. Managua: Fundación Vida, 2001.

Gámez, José Dolores. Catecismo de historia patria. Sin datos. 1894

Gámez, José Dolores. Catecismo de historia de Centroamérica. Managua: Tipografía Nacional, 1900.

Gould, Jeffre L. “”Vana Ilusióni” The Highlands Indian and the Myth of Nicaragua Mestiza”, en Aviva Chomsky y Aldo Lauria-Santiago (editores) Identity and Struggle at the Margins of the Nation-State. The laboring Peoples of Central America and the Hispanic Caribbean. North Carolina, Duke University Press, 1998.

Jenkins Molieri, Jorge. El desafío indígena en Nicaragua: el caso de los mískitos. Managua: Editorial Vanguardia, 1986.

Kinloch Tijerino, Frances. Nicaragua, identidad y cultura política. 1821-1858. Managua: Banco Central de Nicaragua, 1999.

Kinloch Tijerino, Frances. “La idea de nación en la Nicaragua de 1858”, en Memorias del IV Simposio Panamericano de Historia. México: Comisión de Historia - Instituto Panamericano de Geografía e Historia, 2001.

Lévy, Pablo. Notas geográficas y económicas sobre la República de Nicaragua. Managua: Fondo de Promoción Cultural Banco de América, 1976. 
Molina Argüello, Carlos. La enseñanza de la historia en Nicaragua. México: Comisión de Historia - Instituto Panamericano de Geografía e Historia, 1953.

Ortega Arancibia, Francisco. Cuarenta años de Historia de Nicaragua 1838-1878. Managua: Fondo de Promoción Cultural Banco de América, 1974.

Pereira y Castellón, Félix. Cartilla de la Historia de Nicaragua para uso de los niños hijos de obreros. León, Nicaragua: sin datos, 1928.

Portas, Bernardo. Compendio de Historia de Nicaragua. Managua: Tipografía y Encuadernaciones Nacionales, 1918.

Pozas, Víctor S. La revolución sandinista 1979-1988. Madrid: Editorial Revolución, 1988.

Rodríguez Rosales, Isolda. La educación durante el liberalismo. Nicaragua: 1892-1909. Managua: Editorial Banic, 1998.

Rodríguez Rosales, Isolda. Historia de la educación en Nicaragua. Restauración conservadora 1910-1930. Managua: Editorial Hispamer, 2005.

Romero, Jilma (coord). Historia de Nicaragua. Managua: Universidad Nacional Autónoma de Nicaragua, 2002.

Sarlo, Beatriz. La máquina cultural. Maestras, traductores y vanguardistas. Buenos Aires: Ariel, 1998.

Selser, Gregorio. La restauración conservadora y la gesta de Benjamín Zeledón. Nicaragua-Usa 1909-1916. Managua: Aldilá Editor. 1998.

Walter, Knut. El régimen de Anastasio Somoza 1936-1956. Managua: Instituto de Historia de Nicaragua y Centroamérica-Universidad Centroamericana, 2004. 